\title{
A Systems Biological Perspective of Cellular Stress-Directed Programmed Cell Death
}

\author{
Orsolya Kapuy*, Beáta Lizák, Ibolya Stiller, Gábor Bánhegyi \\ Department of Medical Chemistry, Molecular Biology and Pathobiochemistry, Semmelweis University, \\ Budapest, Hungary \\ Email: ${ }^{*}$ kapuy.orsolya@med.semmelweis-univ.hu
}

Received 14 February 2014; revised 3 March 2014; accepted 10 March 2014

Copyright (C) 2014 by authors and Scientific Research Publishing Inc.

This work is licensed under the Creative Commons Attribution International License (CC BY). http://creativecommons.org/licenses/by/4.0/

CC) (i) Open Access

\begin{abstract}
Each eukaryotic cell of multicellular organisms must be able to maintain its integrity by sensing both external and internal stimuli. The primary goal of the generated response mechanism is to drive back the system to the former or to a new homeostatic state. Moreover, the response has to provide an accurate survival-or-death decision to avoid any "misunderstanding" and its unwanted consequences. New data revealed that a systems-level crosstalk of molecular networks has an essential role in achieving the correct characteristic of the response. Although many molecular components of these processes already have been revealed, several elements and regulatory connections of crosstalk are still missing. These "gaps" of the complex control networks make hardly impossible to present comprehensive models. Therefore we approach the questions from a systems biology aspect by combining the experimental results with the special technique of mathematical modelling. In this short report we discuss some novel and preliminary data gained by this approach on the crosstalk between life and death decisions under cellular stress, to get a systems biological view of these networks.
\end{abstract}

\section{Keywords}

Systems Biology; Cellular Stress; Autophagy; Apoptosis; Crosstalk; Mathematical Modelling

\section{Introduction}

For delicate cellular balance sustained by various signal transduction pathways and control elements, the most important criteria are that adequate answers must be formulated if and only if stimuli exist [1]. To understand this process, let's imagine a regulating system for a jet plane fully wired via a lots of switches and arms, while

"Corresponding author. 
each task (e.g. landing, lightening) is directed by a well-defined control module. The regulatory modules of cellular network contain a variety of enzyme proteins which can influence the activity of other proteins in positive or negative direction. The dynamic features of the response mechanism can be described by signal-response curves [1]. Regulatory networks implementing decision making process can be mainly characterized with two different phenomena. In one case a network is designed for broad dynamic ranges and proportionate responses [2]. This corresponds to that feature of a jet plane, when e.g. the pilot can choose the height of the plane above mean sea level continuously in a broad range during flight. According to the wiring of the regulatory elements linear, hyperbolic or sigmoid signal-response curves can be generated [1] [3] (Figure 1(a), upper panel). In the other case, the cellular decision making occurs in a binary way with two discrete stable states separated by an unstable one [3] (Figure 1(a), lower panel). Using our metaphor of jet plane, this can be symbolized for e.g. with the two separated states of the wheels (IN or OUT). These discontinuous switch-like characteristic results in an abrupt change as signal level reaches a critical threshold value in either direction (see grey dotted arrows on Figure 1(a), lower panel). The key regulatory components of the cellular signalling networks are generally wired with positive feedback loops (either mutual activation or antagonism) to achieve all-or-none decision [4]-[6]. Depending on the feature of the control network, the switch can be reversible or irreversible. Reversible response mechanism assumes that the system is able to switch off sensing the elimination of the signal, while one-way switch guarantees that the response element remain in its active state even if the stimuli get disappeared [1] (see black dotted arrow on Figure 1(a), lower panel).

\section{The Characteristic Features of Cellular Life-or-Death Decision}

Usually the cellular decision making process between life and death can be described with an all-or-none characteristic. The suicide network of apoptosis, as a type of cell death mechanisms has been extensively revealed; nowadays plenty of articles are focusing on the cellular self-eating driven by autophagy, too. Since the huge numbers of the regulatory components and the complexity of their connections make hardly impossible to understand the characteristic behaviour of these mechanisms [7], therefore a novel approach called systems biology gets widely used which involves both experimental techniques and mathematical modelling [8] [9].

\subsection{Committing Suicide by Apoptosis}

Apoptosis removes aberrant or damaged cells, but it also has an important role in eliminating cells during embryonic development and immune system maturation [10]. Apoptotic cell death can be easily recognized by cellular shrinkage, mitochondrial permeabilization and DNA fragmentation [11]. The programmed cell death driven by apoptosis has two characteristic rules: 1) cell death response must not be triggered by week signals and 2) once apoptosis is triggered cells should commit to finish the process (i.e. point-of-no-return) [12]. It is very important that the cell must not hesitate on the borderline of two well-separated states (i.e. ON and OFF state of apoptosis); otherwise not only a wrong decision, but also a not finished process might be fatal as well [1] [13]. This is based on principles similar to the take-off decision points of jet planes. The plane is not allowed to start taking-off before reaching the crucial velocity of the plane on the runway. In addition, the pilot cannot break the taking off either after induction, otherwise the plane would crash. Therefore the two states of the plane (grounded and took off) are separated with a discontinuous step. While the pilot is reminded by certain signs of instruments, in cells there are the control modules, which meet the obligation for apoptosis following the realization of the level of cellular stress.

\subsection{Autophagy-Dependent Self-Cannibalism}

Another type of programmed cell death is autophagy. Autophagy is an evolutionary conserved cellular digestive process whereby cytosolic contents are sequestered in vesicles and delivered to the lysosome for degradation [14] [15]. The basic level of autophagy is essential for housekeeping while increased level of autophagic process plays a key role in cellular differentiation, growth control, cell defence, tissue remodelling and different type of cellular stresses (e.g. hypoxia) [14] [16]. Down-regulation of the key sensor of both growth factors and nutrient availability (so called mTOR pathway) results in induction of autophagy, too [17]. Therefore autophagy is not really a self-killing mechanism, rather a cytoprotective process of cells found in eukaryotes from yeast to mammalians [16] [18] [19]. 


\section{The Importance of Crosstalk between Autophagy and Apoptosis}

The control network of both autophagy and apoptosis is complex. In addition, an increasing number of novel results confirm that these two mechanisms are not independent from each other; rather they are connected at various regulatory levels generating crosstalk between them [20]-[22]. While autophagy usually results in survival, apoptosis induces cell death, therefore their crosstalk seems to have an essential role in achieving a well-bal- anced cellular response reacting to various stress signals (e.g. endoplasmic reticulum (ER) stress, nutrient deprivation) [22].

Although an increasing number of experimental data analyses the key components of this crosstalk, relatively few data are focusing on exploring the dynamical features of the crosstalk of the system. Nevertheless it is really an interesting question how the regulatory network can choose between autophagy-dependent survival and apoptosis-induced cell death in respect with stress signal. It has already been proven that autophagy precedes apoptosis during cellular stress [23], but how the system switches between them is still a mystery.

\subsection{The Signal-Response Profile of Both Autophagy and Apoptosis under Cellular Stress}

Based on experimental data our systems biological analysis has shown recently the crucial feedback loops of the regulatory network participating in decision-making process between the life and death [13]. The different components of the regulatory networks were sorted into three groups, called crosstalk element, autophagy inducer and apoptosis inducer and they were connected with crucial feedback loops (Figure 1(b)). By definition autophagy inducers (such as Beclin1, Ambra1, Atg3, Atg4, Atg5) are able to promote the self-eating process, while apoptosis inducers (e.g. Caspase-3, Caspase-6, Caspase-8, Calpain) enhance apoptotic cell death. Experimental data are suggested that cFLIP, vFLIP, $\mathrm{Bcl} 2$ and $\mathrm{Bcl}_{\mathrm{XL}}$ have a regulatory effect on both autophagy and apoptosis inducers [22]. Therefore these molecules are considered as crosstalk elements. Our analysis suggests that the crosstalk element is able to act negatively on both autophagy and apoptosis resulting in a direct link be-

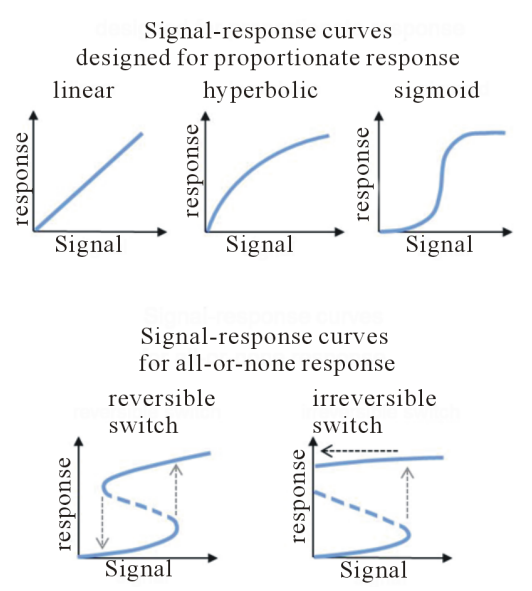

(a)

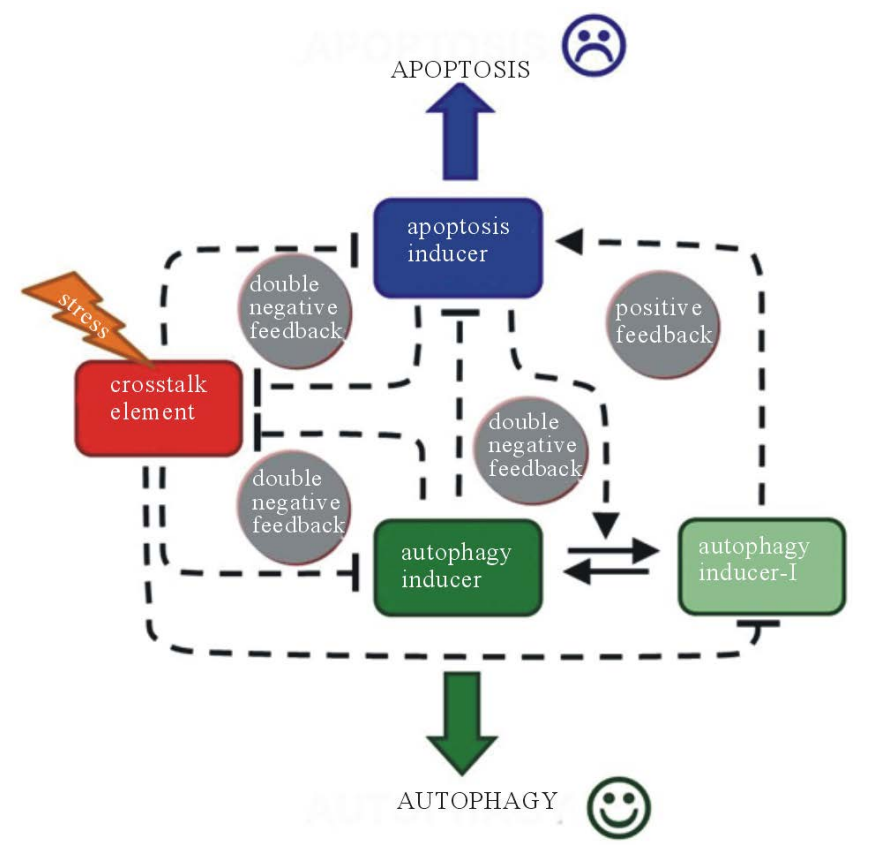

(b)

Figure 1. Studying cellular decision making process. (a) The possible signal-response curves designed for proportionate response (upper panel) and for all-or-none response (lower panel). Solid lines denote stable states, while dashed line presents the unstable ones. (b) The wiring diagram and the key network motifs of autophagy-apoptosis crosstalk. The active forms of the elements are grouped in dark-coloured boxes, while light-coloured box denotes the apoptosis inducer-cleaved form. The experimentally proved regulatory connections between molecules are presented by dashed lines, while solid arrows show biochemical reactions. Blocked end lines denote inhitition. 
tween the two mechanisms [13]. However, autophagy and apoptosis inducers are antagonists generating a mutual exclusion. This opposition in itself provides a peculiar regulatory motif, so-called double negative feedback in the system. In addition, there is a positive feedback between apoptosis inducer and inactive autophagy inducer. The apoptosis inducer-dependent inactive form of autophagy directly promotes apoptotic cell death (Figure 1(b)).

Plotting the signal-response curve of both autophagy and apoptosis inducers, it could be seen that autophagy inducer follows a sigmoid activation profile, while apoptosis inducer has a switch-like characteristic according to the level of cellular stress (Figure 2(a), lower panel). Under normal physiological conditions autophagy has a basic level, which is high enough to block the apoptosis inducer. With respect to stress autophagy inducer quickly gets activated through down-regulation of crosstalk element, meanwhile apoptosis inducer remains fully inactive. Although crosstalk element has almost been disappeared promoting apoptosis inducer the sigmoid increase of autophagy inducer is able to down-regulate apoptotic cell death (Figure 2(a)). However reaching a given threshold value of the signal (i.e. when cellular stress becomes excessive) apoptosis inducer gets activated immediately and switches off the autophagic process (Figure 2(a), lower panel). The signal-response curve of apoptosis inducer has two distinguished stable states. In one state (at normal physiological conditions and at low level of cellular stress) apoptosis inducer is OFF, while autophagy works with different intensity depending on the level of cellular stress. In the other state (at severe cellular stress) apoptosis inducer switches ON, autophagy gets immediately inactivated due to the mutual antagonism (Figure 2(a)).

Consistently with our theoretical interpretation, Xu et al. confirmed that autophagy preceded apoptosis in response to different cellular stress levels. They used single cell live imaging to demonstrate the dynamic characteristic of both autophagy and apoptosis mechanisms [24]. They also showed that autophagy induction is unimodal, while apoptosis induction has an all-or-none bimodal pattern [24]. Adding different amount of staurosporin they found that (similarly to the theoretical computer simulations) the level of cellular stress has to reach a threshold to switch on the apoptotic process. Otherwise autophagy wins against apoptosis and the self-killing pathway will be only transiently activated, which would not be sufficient to the fatal cellular decision. Unsurprisingly, inhibition of autophagy by mutating any key components of the survival process results in premature activation of apoptosis in response to cellular stress [24].

\subsection{The Dynamic Properties of Crosstalk between Autophagy and Apoptosis}

The mutual antagonism between autophagy and apoptosis ensures a precise decision between life and death depending on the level of cellular stress, but one question has not been answered yet; namely, what is the mechanism to control that first autophagy is activated under cellular stress then the cell later allows the suicide process?

To our knowledge the crosstalk element has a crucial role to render the correct order of autophagy dependent survival and apoptotic cell death under cellular stress. According to the effect of crosstalk element on programmed cell death mechanisms there are two possibilities. In the first case the crosstalk element (e.g. Bcl2) inhibits both autophagy and apoptosis inducers [13]. When cellular stress is imposed the activity of autophagy inducer would become fully active earlier than the apoptosis inducer, while the level of crosstalk element gets decreased by cellular stress (Figure 2(a), lower panel). Although the crosstalk element was trying to switch on the apoptosis, the autophagy inducer hindered the cell death process. If the stress situation sustained the apoptosis inducer would be eventually active. In line with this step the autophagy inducer immediately switched off (see Figure 2(a), lower panel). Even if the crosstalk element is completely diminished afterwards, the survival mechanisms could not function any more, i.e. the whole energy of the cell is devoted to execute suicide.

In an alternative model we claim that the crosstalk element is the so-called unfolded protein response (UPR) induced by ER stress. Cellular stress acts positively on crosstalk element resulting in its hyperbolic activation (Figure 2(b)). ER stress via UPR activates both autophagy and apoptosis. Autophagy came first, while apoptosis induction requires higher concentration of cellular stress to down-regulate the survival process via a double negative feedback loop (Figure 2(b)). The systems-level analysis shows that the crosstalk element has an important role in ensuring the correct order of the two mechanisms during cellular stress, while the feedback loops between autophagy and apoptosis inducers determine the dynamic profiles of the control system [Kapuy, unpublished results].

Although the underlying mechanism is very similar, different types of cellular stressors are able to trigger 


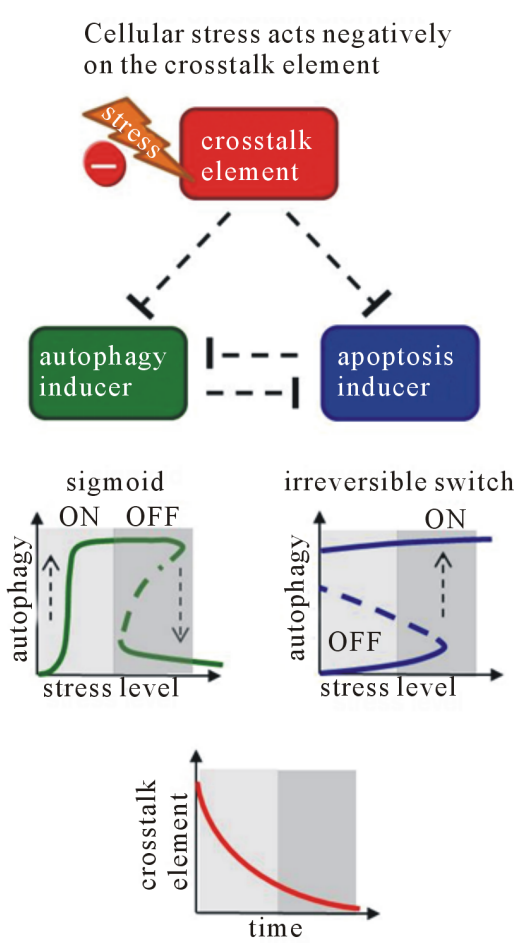

(a)
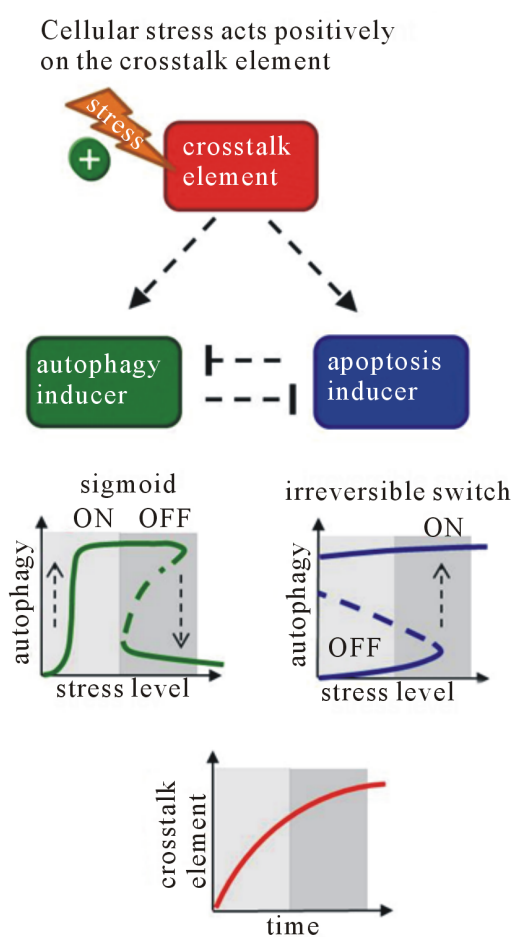

(b)

Figure 2. The dynamic characteristic of life-or-death decision under celullar stress. The simplified wiring diagram of autophagy-apoptosis crosstalk (upper panel), the signal-response curve of both autophagy and apoptosis and the time course of crosstalk element (lower panel) are presented, where (a) crosstalk element inhibits both autophagy and apoptosis inducers; (b) crosstalk element activates both autophagy and apoptosis inducers. Upper panel: the autophagy inducer, the apoptosis inducer and the crosstalk element are depicted by isolated green, blue and red boxes, respectively. Dashed lines show how the elements can influence each other. Lower panel: solid lines denote stable states, while dashed line denotes the unstable state. Light grey background refers to tolerable stress, while dark grey background shows excessive level of cellular stress.

various components of the upstream regulators. For example, in ER stress mTOR pathways have a key role to promote apoptosis via down-regulating autophagy [25]-[27]. A double negative feedback loop was experimentally confirmed between autophagy and mTOR, while apoptosis activates mTOR supposing a positive linkage between them [25]. According to the experimental data it was suggested with theoretical techniques that both the positive effect on apoptosis inducer and the negative effect on autophagy inducer by mTOR is essential to expand the window of survival when mTOR is inhibited by rapamycin pre-treatment [Kapuy, submitted results]. These extra feedback loops might have a role in securing the robustness of the answer mechanism.

\section{Conclusions}

Recent experimental data [24] together with our analysis [13] showed that the systems-level double negative and positive feedbacks guarantee the precisely executed life-or-death decision under cellular stress. A crosstalk between cytoprotective autophagy and self-killing apoptosis ensures that the sigmoid induction of autophagy always precedes the switch-like activation of cell death even at excessive level of cellular stress. This process is controlled by various crosstalk elements (e.g. Bcl2). Our preliminary results suggest that crosstalk elements can act on both autophagy and apoptosis inducers positively and negatively, but the strength of these regulatory connections is crucial to render a transient activation for autophagy, even under fatal cellular stress. Compartmentation of the crosstalk element can also help to maintain the proper order of autophagy and apoptotic cell death [21], while extra feedback loops (e.g. mTOR-UPR crosstalk) might have a role in securing the robustness of the answer mechanism. However, the exploration of these systems-level feedbacks will be a challenge for fu- 
ture research.

The number of elements participating in the crosstalk between autophagic and apoptotic pathways is continuously growing. Despite the experimental efforts, the network of survival-or-death decision-making is hardly understood. These circumstances necessitate the use of alternative, non-experimental approaches. Systems biology offers the possibility to develop models with a predictive value. These kinds of models have been successfully applied for the description of different parts of the autophagic and apoptotic pathways. However, a comprehensive model for the whole network of crosstalking is painstakingly missing due to the very high number of nodes and edges in the complete system. Thus, a royal road to understand the process of decision-making can be the development of comprehensive but less detailed models. Since autophagy-apoptosis crosstalk induced by cellular stress is tightly connected to a variety of diseases, such as diabetes, various liver and neurodegenerative diseases (e.g. Parkinson's or Huntington's diseases), the long-term significance of these works implies medical purposes.

\section{Acknowledgements}

This work was supported by OTKA-PD Grant from the Hungarian Scientific Research Fund (OTKA-PD 104110). OK is thankful to Bolyai Research Scholarship of the Hungarian Academy of Sciences. We thank Dr. Enuo He for critically reading the manuscript.

\section{References}

[1] Tyson, J.J., Chen, K.C. and Novak, B. (2003) Sniffers, Buzzers, Toggles and Blinkers: Dynamics of Regulatory and Signaling Pathways in the Cell. Current Opinion in Cell Biology, 15, 221-231. http://dx.doi.org/10.1016/S0955-0674(03)00017-6

[2] Biggar, S.R. and Crabtree, G.R. (2001) Cell Signaling Can Direct Either Binary or Graded Transcriptional Responses. The EMBO Journal, 20, 3167-3176. http://dx.doi.org/10.1093/emboj/20.12.3167

[3] Tyson, J.J. and Novak, B. (2010) Functional Motifs in Biochemical Reaction Networks. Annual Review of Physical Chemistry, 61, 219-240. http://dx.doi.org/10.1146/annurev.physchem.012809.103457

[4] Tyson, J.J., Chen, K. and Novak, B. (2001) Network Dynamics and Cell Physiology. Nature Reviews Molecular Cell Biology, 2, 908- 916. http://dx.doi.org/10.1038/35103078

[5] Tyson, J.J., Csikasz-Nagy, A. and Novak, B. (2002) The Dynamics of Cell Cycle Regulation. BioEssays, 24, 10951109. http://dx.doi.org/10.1002/bies.10191

[6] Novak, B., et al. (2010) Regulated Protein Kinases and Phosphatases in Cell Cycle Decisions. Current Opinion in Cell Biology, 22, 801-808. http://dx.doi.org/10.1016/j.ceb.2010.07.001

[7] Tyson, J.J., et al. (2011) Dynamic Modelling of Oestrogen Signalling and Cell Fate in Breast Cancer Cells. Nature Reviews Cancer, 11, 523-532. http://dx.doi.org/10.1038/nrc3081

[8] Arkin, A.P. and Schaffer, D.V. (2011) Network News: Innovations in 21st Century Systems Biology. Cell, 144, 844849. http://dx.doi.org/10.1016/j.cell.2011.03.008

[9] Nurse, P. and Hayles, J. (2011) The Cell in an Era of Systems Biology. Cell, 144, 850-854. http://dx.doi.org/10.1016/j.cell.2011.02.045

[10] Taylor, R.C., Cullen, S.P. and Martin, S.J. (2008) Apoptosis: Controlled Demolition at the Cellular Level. Nature Reviews Molecular Cell Biology, 9, 231-241. http://dx.doi.org/10.1038/nrm2312

[11] Elmore, S. (2007) Apoptosis: A Review of Programmed Cell Death. Toxicologic Pathology, 35, 495-516. http://dx.doi.org/10.1080/01926230701320337

[12] Zhang, T., Brazhnik, P. and Tyson, J.J. (2009) Computational Analysis of Dynamical Responses to the Intrinsic Pathway of Programmed Cell Death. Biophysical Journal, 97, 415-434. http://dx.doi.org/10.1016/j.bpj.2009.04.053

[13] Kapuy, O., et al. (2013) A Cellular Stress-Directed Bistable Switch Controls the Crosstalk between Autophagy and Apoptosis. Molecular BioSystems, 9, 296-306. http://dx.doi.org/10.1039/c2mb25261a

[14] Levine, B. and Kroemer, G. (2008) Autophagy in the Pathogenesis of Disease. Cell, 132, 27-42. http://dx.doi.org/10.1016/j.cell.2007.12.018

[15] Wirawan, E., et al. (2012) Autophagy: For Better or for Worse. Cell Research, 22, 43-61. http://dx.doi.org/10.1038/cr.2011.152

[16] Ravikumar, B., et al. (2010) Regulation of Mammalian Autophagy in Physiology and Pathophysiology. Physiological Reviews, 90, 1383-1435. http://dx.doi.org/10.1152/physrev.00030.2009 
[17] Hay, N. and N. Sonenberg (2004) Upstream and Downstream of mTOR. Genes \& Development, 18, 1926-1945. http://dx.doi.org/10.1101/gad.1212704

[18] Wang, S.Y., et al. (2011) Core Signaling Pathways of Survival/Death in Autophagy-Related Cancer Networks. The International Journal of Biochemistry \& Cell Biology, 43, 1263-1266. http://dx.doi.org/10.1016/j.biocel.2011.05.010

[19] Levine, B. and Klionsky, D.J. (2004) Development by Self-Digestion: Molecular Mechanisms and Biological Functions of Autophagy. Developmental Cell, 6, 463-477. http://dx.doi.org/10.1016/S1534-5807(04)00099-1

[20] Maiuri, M.C., et al. (2007) Self-Eating and Self-Killing: Crosstalk between Autophagy and Apoptosis. Nat Rev Mol Cell Biol, 8, 741-752. http://dx.doi.org/10.1038/nrm2239

[21] Eisenberg-Lerner, A., et al. (2009) Life and Death Partners: Apoptosis, Autophagy and the Cross-Talk between Them. Cell Death Differ, 16, 966-975. http://dx.doi.org/10.1038/cdd.2009.33

[22] Gordy, C. and He, Y.W. (2012) The Crosstalk between Autophagy and Apoptosis: Where Does This lead? Protein Cell, 3, 17-27. http://dx.doi.org/10.1007/s13238-011-1127-x

[23] Ogata, M., et al. (2006) Autophagy Is Activated for Cell Survival after Endoplasmic Reticulum Stress. Molecular and Cellular Biology, 26, 9220-9231. http://dx.doi.org/10.1128/MCB.01453-06

[24] Xu, Y., Yuan, J. and Lipinski, M.M. (2013) Live Imaging and Single-Cell Analysis Reveal Differential Dynamics of Autophagy and Apoptosis. Autophagy, 9, 1418-1430. http://dx.doi.org/10.4161/auto.25080

[25] Appenzeller-Herzog, C. and Hall, M.N. (2012) Bidirectional Crosstalk between Endoplasmic Reticulum Stress and mTOR Signaling. Trends in Cell Biology, 22, 274-282. http://dx.doi.org/10.1016/j.tcb.2012.02.006

[26] Walter, P. and Ron, D. (2011) The Unfolded Protein Response: From Stress Pathway to Homeostatic Regulation. Science, 334, 1081-1086. http://dx.doi.org/10.1126/science.1209038

[27] Watanabe, R., Wei, L. and Huang, J. (2011) mTOR Signaling, Function, Novel Inhibitors, and Therapeutic Targets. Journal of Nuclear Medicine, 52, 497-500. 